\title{
Ultrasonic Strain Measurements of Tendon
}

\author{
Pai-Chi $\mathrm{Li}^{1}$, Po-Lin Kuo ${ }^{2}$ and Chia-Tung Shun ${ }^{3}$ \\ ${ }^{1}$ Department of Electrical Engineering, National Taiwan University \\ ${ }^{2}$ Department of Physical Medicine and Rehabilitation, National Taiwan University Hospital \\ ${ }^{3}$ Department of Pathology, National Taiwan University Hospital \\ Taipei, Taiwan, R.O.C.
}

Abstract - Elasticity imaging has gained wide interest in medical ultrasound. In rehabilitative medicine, such a tool may be of great clinical importance since it is capable of directly assessing the mechanical properties of tendon. A critical component in elasticity imaging is estimation of the strain fields. In this study, axial strain fields of rabbit Achilles tendons were measured in vitro. Strain measurements were also correlated with conventional B-mode sonograms and histological micrographs. Results indicated that the strain measurements had reasonable agreement with histological examinations and provided better tissue differentiation than conventional B-mode imaging. For a given tendon, the strain values corresponding to different tissue layers were easily differentiated. Such differences, however, were not always present in the sonograms. An elasticity reconstruction approach based on axial strain measurements was also briefly discussed.

\section{INTRODUCTION}

A main function of tendons is to assist in concentrating the pull of the muscle on a small area of bone. The strength and stiffness of a tendon are provided by the cross-links between numerous collagen fibers that are parallel to its longitudinal axis. Since hematoma and inflammatory cell infiltration disrupt the arrangement of collagen fibers, the strength of a tendon tends to degrade when it is injured or inflamed [1]: The primary purpose of rehabilitation is to restore the mechanical functions of an injured or inflamed tendon. To monitor the tendon functions during the rehabilitation process, in vivo non-invasive methods, such as ultrasound, are thus of great clinical importance.
A potential application of ultrasound in this area is elasticity imaging [2-7]. Conventional B-mode imaging is used to produce pictures of anatomical structures. Ultrasonic elasticity imaging, on the other hand, is used to quantitatively assess the elastic properties of biological tissue. Such an imaging method typically involves externally applied forces and reconstruction of strain fields. Reconstruction of elastic properties generally constitutes two steps. The first step involves estimating the strain fields from the ultrasound data acquired pre- and post-compression. The second step is to find the distribution of elastic modulus given the estimated strain fields. The primary purpose of this study is regarding the first step of elasticity imaging. Feasibility of estimating the strain fields of tendons using ultrasound will be studied. Furthermore, the distribution of strain fields will be compared to B-mode sonograms and correlated with histology. The paper concludes with a brief discussion on a potential approach for elasticity reconstruction based on axial strain measurements.

\section{MATERIALS AND METHODS}

Achilles tendons from adult New Zealand white rabbits were used in this study. The animals were purchased from the National Animal Center and were housed in animal rooms with good environmental control. For each rabbit, one randomly selected hind limb was included in the inflamed group and the other hind limb was included in the healthy group. The tendons in the inflamed group were prepared based on the following procedures. First, general anesthesia was applied with $5 \mathrm{mg}$ phenothiazine and $25 \mathrm{mg}$ ketamine per $\mathrm{kg}$ body weight intramuscularly. Next, the skin overlying the Achilles tendon was 
shaved, scrubbed and anesthetized locally with $20 \mathrm{mg}$ lidocaine. A longitudinal incision was made lateral to the visible outline of the tendon. By blunt dissection, the Achilles tendons in the inflamed group were then exposed, isolated from the adjoining tissue and tightened circumferentially on the rostral and caudal sides with nylon sutures. The rostral site was near the tendon and muscle belly junction. The caudal site was near the tendon and calcaneus junction. The sutures were tightened firmly in order to simulate tendon ischemia. The skin was then closed. No particular treatments were done to the tendons in the healthy group.

After the rabbits regained consciousness, they were housed one per standard rabbit cage and raised as normal. Food and water were available ad libitum. The use and care of animals were in accordance with the principles of the National Animal Center of R.O.C. We have found empirically that a period of 10 days is needed to ensure obvious inflammation for ischemic tendon after wound closure. At that time, Achilles tendons in both the inflamed group and the healthy group were harvested. After excision, each sample was then immediately immersed in the center of a gelatin based phantom and was paired with its opposite side.

The image phantoms were constructed from agar powder ( $5 \%$ by weight) with similar stiffness to each other. The phantoms were cylindrical with a diameter of $114.5 \mathrm{~mm}$ and a length of $45.0 \mathrm{~mm}$. When making the phantoms, the following steps were taken to keep the longitudinal axis of the tendon sample parallel to the surface of the phantom. First, gel solution was poured into the mold until a height of $20 \mathrm{~mm}$ was reached. Next, as the gel surface congealed, the tendon sample was put on the center of this surface and additional gel solution was poured into the remaining mold space. All the above procedures were performed on a leveled desk. After the completion of the above procedures, the phantoms were stored at $10^{\circ} \mathrm{C}$ and ultrasound data were collected within 12 hours.

An unfocused, $5 \mathrm{MHz}$, circular transducer (Panametrics V110, USA) with a diameter of $7.9 \mathrm{~mm}$ was attached to a hole in an acrylic plate with the direction of the ultrasound beam perpendicular to the plate. The distance between the sample and the transducer was set at $20 \mathrm{~mm}$ to reduce near field effects and to avoid transducer ring-down artifacts. Prior to each measurement, the acrylic base was leveled such that direction of the gravitational force applied at the top of the phantom was parallel to the direction of the axial displacement. The transducer was connected to an ultrasonic pulser/receiver (Panametrics 5072PR, USA) for A-scan data acquisition. Maximum output power was used. Attenuation and damping settings, on the other hand, were adjusted for each measurement to achieve sufficient signal-to-noise ratios. A digital oscilloscope (Lecroy 9314L, Switzerland) was used to digitize the received signal at $100 \mathrm{MHz}$.

Ultrasonic coupling gel was applied on the transducer face for efficient acoustic transmission. The phantom was positioned such that the tendon intersected with the center of the ultrasound beam. The phantom was compressed by adding weights to the top surface (i.e., the weight side). A $0.5 \mathrm{~kg}$ weight was initially used to pre-load the phantom and to ensure good contact at the transducer-phantom boundary. After the phantom was pre-loaded, additional weights were applied with a $0.5 \mathrm{~kg}$ increment. A baseband speckle tracking algorithm was used to estimate displacement and strain [3]. We have found that a $0.5 \mathrm{~kg}$ increment was able to provide sufficient displacements measurable by the speckle tracking technique while avoiding signal decorrelation resulting from changes in scatterer distribution. After each increment, returning echoes were sampled using a digital oscilloscope. The digitized A-scan signals were then transferred to a personal computer via a GPIB port for off-line signal processing. The cross-sectional area of the weights was the same as that of the phantom. In other words, the top surface of the phantom was fully covered. Additionally, a plastic cover was placed between the weights and the phantom to ensure uniform external stress distribution at the top surface. The experiments were performed at room temperature.

\section{RESULTS}

Eight tendon samples were used in this study. Figure 1 shows B-mode sonograms and estimated strain fields of two of the eight samples. The two samples were from the same rabbit. Panel $A$ is the B-mode sonogram of the healthy tendon in the transverse view and panel $B$ shows the longitudinal view. The tendon appears as a homogeneous, hyper-echoic region in both views. Note that the sonograms and strain field in Fig. 1 are oriented in 
such a way that the bottom of sonograms and the left of strain field correspond to the transducer side of phantom. Although the tendon appears homogeneous in B-mode, the transverse strain shown in panel $\mathrm{E}$ varies over a wide range. The strain variations can be explained by the micrograph shown in Fig. 2 . The micrograph shows that the specimen consists of muscle tissue in the center and bundles of collagen fibers spreading near the edges. Note that the central muscle tissue is about 4 times as thick as the peripheral collagen fiber layer. This ratio is consistent with that shown in the estimated strain field. Although the strain values are clearly different as shown in the panel $\mathrm{E}$ of Fig. 1, the differences between muscle and bundles of collagen fibers are not detectable in B-mode.

Sonograms and strain field of the inflamed sample are shown in panels $\mathrm{C}, \mathrm{D}$ and $\mathrm{F}$. The sonograms for the inflamed sample are similar to those of healthy sample. On the other hand, the strain variation of inflamed sample is larger than that of healthy sample. Fig. 3 shows the histology of the inflamed sample. The micrograph indicates that the area in the middle $2 \mathrm{~mm}$ corresponds to the high strain region and is filled with granulation tissues associated with necrosis. Note that muscle tissue also
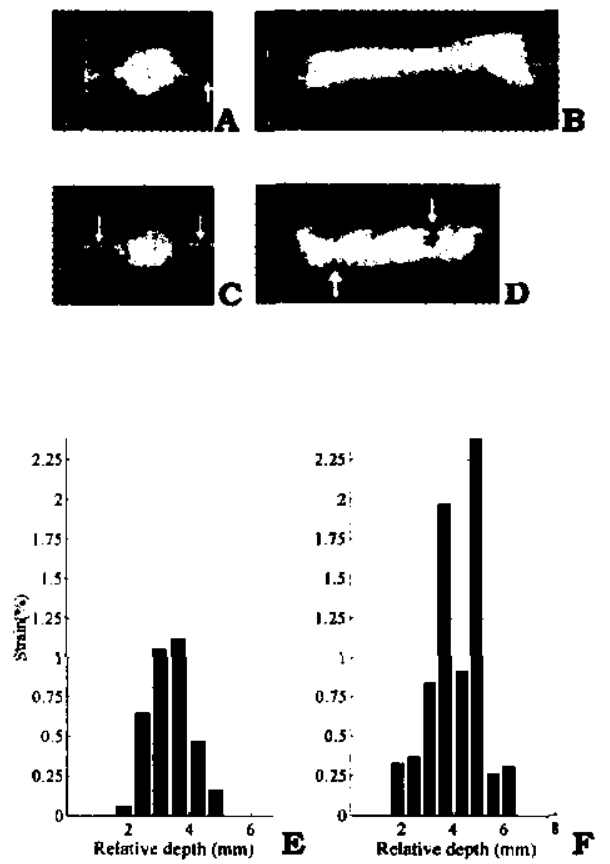

Fig. 1. B-mode sonograms (A: transverse, B: longitudinal) and strain field (E) of the healthy tendon. B-mode sonograms (C: transverse, D: longitudinal) and strain field (F) of the healthy tendon. exists among granulation tissues. Hence, the change in tissue type may explain the high strain variation in the middle $2 \mathrm{~mm}$ region .

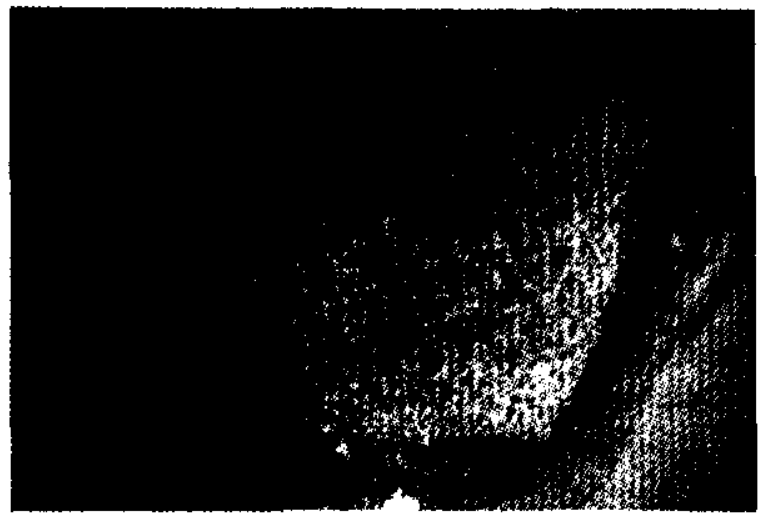

Fig. 2. Micrograph of the healthy sample (M: muscle cell, $\mathrm{C}$ : bundles of collagen fibers).

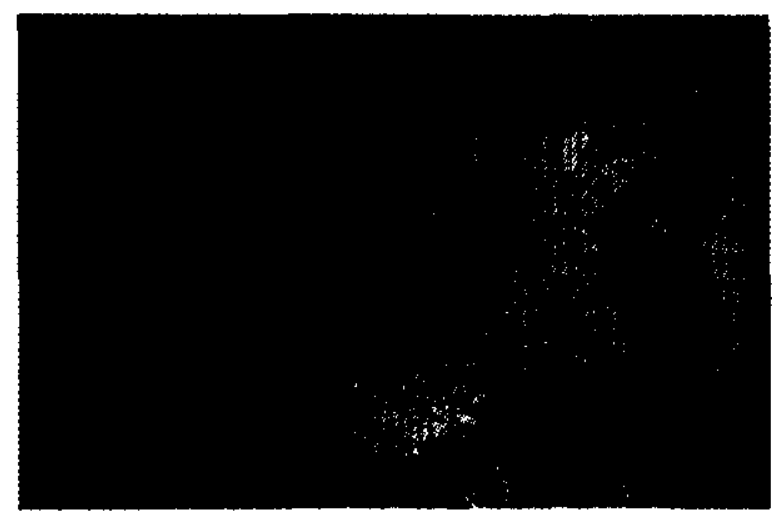

Fig. 3. Micrograph of the inflamed sample (M: muscle cell, C: bundles of collagen fibers, $\mathrm{G}$ : granulation tissue).

\section{DISCUSSION}

In this study, we successfully measured the axial components of transverse strain fields in rabbit's Achilles tendons using the baseband speckle tracking algorithm. Although tendons are generally harder to compress than normal soft tissues, the baseband speckle tracking technique was able to detect displacements as small as a few microns. The strain measurements showed reasonable agreement with histology, whereas the contrast of the sonograms was sometimes insufficient for tissue differentiation. It is concluded that strain imaging has the potential to be a useful tool for evaluating tendon disorders during the rehabilitation process.

Although the axial components of transverse 
strain fields were successfully measured, reconstruction of the elastic modulus requires additional information. Ideally, the elastic modulus can be calculated numerically based on a set of linear equations made from both the static equilibrium equations and the stress-strain constitutive equations. Unfortunately, the stress component cannot be directly measured in most situations. Therefore, alternative information must be provided in order to reconstruct the elastic modulus. Some approaches employ a theoretical model for an isotropic, continuous and almost incompressible medium. The elastic behavior of such a material can thus be completely characterized by the spatial distribution of either the shear modulus or the Young's modulus. Due to the anisotropic nature of tendons, however, new reconstruction algorithms must be employed. For example, Levinson has verified that a transversely isotropic model provided a better fit of elastic constants for muscle than an isotropic model [8]. Such a model may also be applicable to tendons. In general, the stress-strain relationship of a transversely isotropic (hexagonal) material can be represented by the following equation

$$
\left[\begin{array}{l}
T_{1} \\
T_{2} \\
T_{3} \\
T_{4} \\
T_{5} \\
T_{6}
\end{array}\right]=\left[\begin{array}{llllll}
c_{11} & c_{12} & c_{13} & 0 & 0 & 0 \\
c_{12} & c_{11} & c_{13} & 0 & 0 & 0 \\
c_{13} & c_{13} & c_{33} & 0 & 0 & 0 \\
0 & 0 & 0 & c_{44} & 0 & 0 \\
0 & 0 & 0 & 0 & c_{44} & 0 \\
0 & 0 & 0 & 0 & 0 & c_{66}
\end{array}\right]\left[\begin{array}{l}
S_{1} \\
S_{2} \\
S_{3} \\
S_{4} \\
S_{5} \\
S_{6}
\end{array}\right],
$$

where $c_{66}=\left(c_{11}-c_{12}\right) / 2$ and the indices are based on Voigt notation.

Reconstruction of the Young's modulus requires knowledge of five stiffness constants and it is much more complicated than reconstruction in the isotropic case. However, the complexity can be reduced by creating plain strain state. In other words, if the displacements along the longitudinal axis are small compared to the displacements in the transverse plane, the transverse isotropy can be reduced to isotropy in the $2 \mathrm{D}$ transverse plane. Furthermore, assuming that the stiffness inside the tendon is distinctly different from that outside the tendon, the ratio of the Young's moduli inside and outside of the tendon may be found by using a single component of the strain tensor following a procedure similar to the one outlined in [6]. Therefore, relative Young's modulus at particular points on the tendon boundary can be found using the axial strain measurements. However, it has to be pointed out that plain strain state was not achieved for the measurements in this study.

\section{ACKNOWLEDGEMENT}

This work was supported in part by NSC Grant NSC-87-2218-E-002-052.

\section{REFERENCES}

[1] M.I. Mason and H.S. Allen "The rate of healing of tendons: a experimental study of tensile strength". Ann Surg 113, pp. 424-459, 1941.

[2] R.M. Lerner, S.R. Huang and K.J. Parker, "'Sonoelasticity" images derived from ultrasound signals in mechanically vibrated tissues". Ultrasound Med Biol, 16(3) pp.231-239, 1990.

[3] M. O'Donnell, A.R. Skovoroda, B.M. Shapo, S. Emelianov, "Internal displacement and strain imaging using ultrasonic speckle tracking", IEEE Trans on Ultrason Ferroelec Freq Control, 41(3), pp.314-25, 1994.

[4] J. Ophir, I. Céspedes and H. Ponnekanti, "Elastography: a quantitative method for imaging the elasticity of biological tissues", Ultrason Imag 13, pp.111-134, 1991.

[5] A. P. Sarvazyan, "Method and device for shear wave elasticity imaging". US patent number 5,606,971. 1997.

[6] A.R. Skovoroda, S. Emelianov, M. O'Donnell, "Tissue elasticity reconstruction based on ultrasonic displacement and strain images", IEEE Trans Ultrason Ferroelec Freq Control, 42(4), pp.747-765, 1995.

[7] F. Kallel and M. Bertrand, "Tissue elasticity reconstruction using linear perturbation method", IEEE Trans Med Imag, 15(3), pp.209-213, 1996.

[8] S.F. Levison, "Ultrasound propagation in anisotropic soft tissue: the application of linear elasticity theory", J Biomech, 20(3), pp.251-260, 1987. 